
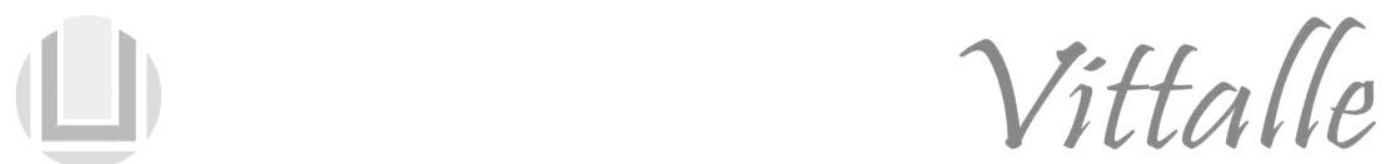

\title{
Tratamento da Tenossinovite de De Quervain por Acupuntura - Relato de Caso
}

\author{
Fernando Mendes Sant'Anna ${ }^{\mathrm{a}, *}$, Vanessa Maia Rangel ${ }^{\mathrm{b}}$, Durval Dionísio Souza Mota ${ }^{\mathrm{b}}$ \\ ${ }^{\text {a}}$ Universidade Federal do Rio de Janeiro, Campus Macaé, Rio de Janeiro, RJ, Brasil \\ Hospital Universitário Antônio Pedro, Niterói, RJ, Brasil. \\ ${ }^{\mathrm{b}}$ Instituto de Saúde e Sociedade da Universidade Federal Fluminense, Niterói, RJ, Brasil
}

\begin{abstract}
Histórico do Artigo
Recebido em:

$06 / 11 / 2017$

Aceito em:

$30 / 03 / 2018$

Palavras chave:

Acupuntura;

tenossinovite de De

Quervain; medicina

tradicional chinesa.
\end{abstract}

Keywords:

Acupuncture;

De Quervain's

tenosynovitis;

traditional chinese

medicine.

\begin{abstract}
RESUMO
A tenossinovite de De Quervain (TDQ), decorrente da inflamação dos tendões dos músculos abdutor longo e extensor curto do polegar, vem aumentando sua incidência, no mundo, nos últimos anos, sendo mais uma dentre as doenças ocupacionais relacionadas ao trabalho. Seu tratamento clássico é realizado através de anti-inflamatórios não esteroides, infiltração de corticoide local e cirurgia reparadora. A acupuntura desponta como alternativa terapêutica válida e de baixo custo para tratamento dessa e de outras síndromes, dentro das práticas integrativas e complementares. Relatamos, neste artigo, um caso de TDQ encaminhado ao nosso ambulatório, ao qual o tratamento por acupuntura, dentro dos princípios da medicina tradicional chinesa (MTC), propiciou breve retorno ao trabalho e completa resolução dos sintomas em curto prazo de tempo. A paciente foi tratada por 5 sessões consecutivas, semanais, e os pontos utilizados foram: IG4, IG5, P7, F3 e Yintang, além de pontos Ah shi.
\end{abstract}

Treatment of De Quervain's Tenosynovitis by Acupuncture - Case Report

\section{ABSTRACT}

De Quervain's tenosynovitis (DQT), resulting from inflammation of the tendons of the abductor pollicis longus and extensor pollicis brevis muscles, is increasing its incidence in the world in recent years, being another of the occupational illnesses related to work. Its classical treatment is accomplished through non-steroidal anti-inflammatory drugs, local corticosteroid infiltration and reconstructive surgery. Acupuncture stands out as a valid and cost-effective treatment option of this and other syndromes, among the integrative and complementary practices. We report in this article a case of DQT forwarded with outpatient clinic. The patient was treated for 5 sessions, in consecutive weeks, and the points used were: LI4, LI5, LU7, LR3 and Yintang, beside Ah shi points. The results showed the use of acupuncture based on the principles of Traditional Chinese Medicine (TCM) provided brief return to work and complete resolution of symptoms in short period of time.

\section{Introdução}

A tenossinovite do primeiro compartimento extensor do punho é também conhecida como tenossinovite de De Quervain (TDQ), em homenagem ao cirurgião suíço Fritz De Quervain (1). Trata-se, em geral, de mais uma dentre as doenças osteomusculares relacionadas ao trabalho (DORT), cuja incidência vem crescendo no mundo, embora sua causa possa ser multifatorial e envolva também fatores genéticos ligados ao sexo, sendo uma patologia que tem maior incidência entre as mulheres (2).

Essa doença se localiza no primeiro compartimento dorsal do punho. Sob a bainha sinovial trafegam os tendões dos músculos abdutor longo e extensor curto do polegar que, com o tempo, apresentam um processo inflamatório local, que atinge os tecidos sinoviais peritendinosos e os tecidos próprios dos tendões (3). É uma das doenças que mais frequentemente acometem o punho.

\footnotetext{
*Autor correspondente: fmsantanna@gmail.com (F.M. Sant ${ }^{*}$ Anna)
} 
O diagnóstico de TDQ é essencialmente clínico, baseado primariamente no achado de dor puntiforme junto ao processo estiloide do rádio, que piora com a movimentação do punho ou do primeiro quirodáctilo. As duas manobras mais clássicas para diagnosticar TDQ são os testes de Finkelstein (4) e de Eichoff (5), ilustrados na Figura 1.
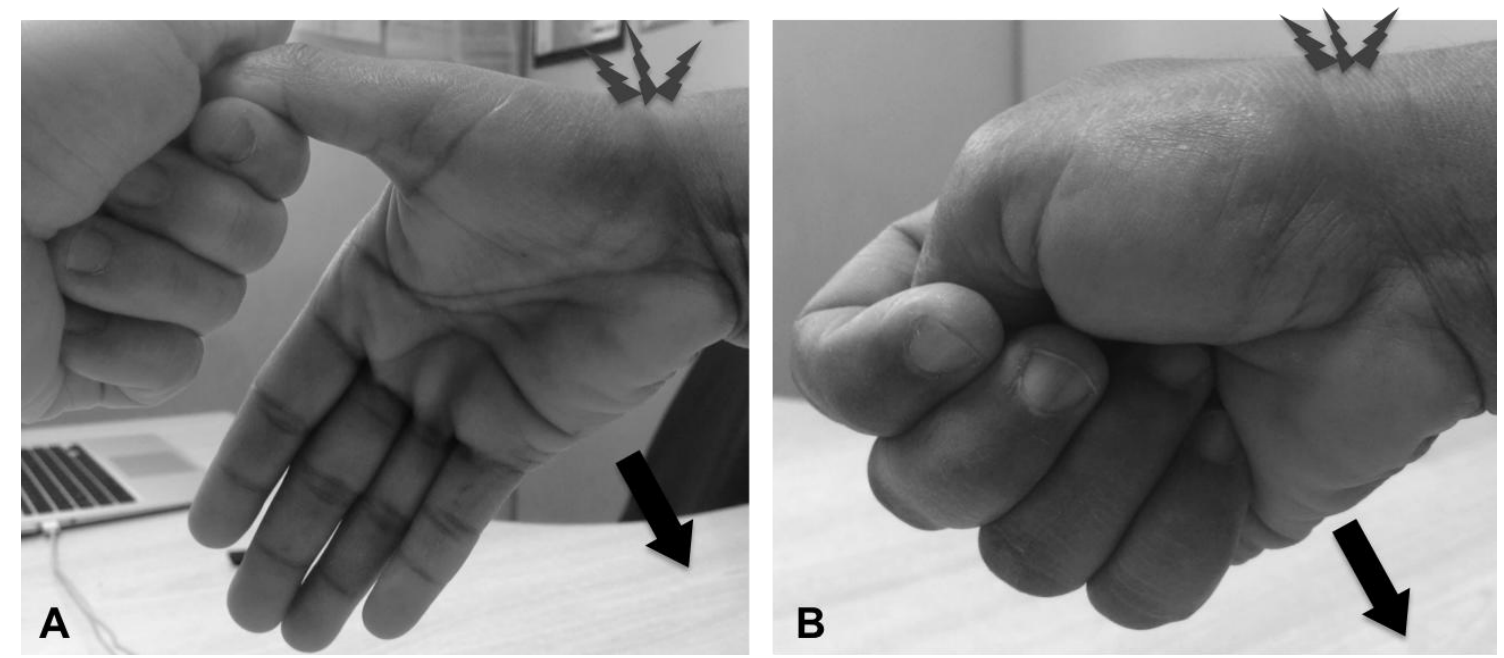

Figura 1: Testes utilizados para diagnóstico clínico de tenossinovite de De Quervain. A: Teste de Finkelstein, mantém-se o polegar estável enquanto se faz o desvio ulnar do carpo. B: Teste de Eichoff, consiste em realizar preensão do polegar em flexão enquanto é realizado o desvio ulnar do carpo. A positividade de ambos os testes se manifesta por dor junto ao processo estiloide do rádio durante o desvio ulnar do carpo.

A acupuntura é um dos braços mais conhecidos da Medicina Tradicional Chinesa (MTC). Consiste no tratamento pela inserção de agulhas em pontos determinados do corpo, seguindo as linhas dos meridianos, que são traçados imaginários distribuídos por todo o corpo $(6,7)$. Por ser de origem empírica, a acupuntura nem sempre teve crédito. Entretanto, trabalhos realizados nas últimas décadas comprovaram a eficácia da técnica no tratamento da dor (8), sendo recomendada pelo National Institutes of Health (NIH) como terapia complementar ou tratamento coadjuvante em programas de controle de dor para vários tipos de doenças (9).

Inicialmente, acreditava-se que os efeitos da acupuntura eram mediados apenas por fibras nervosas mielinizadas A delta, responsáveis pela sensação de picada da agulha e/ou por um mecanismo de controle do portão da dor (gate control), no qual interferem as fibras A beta, de condução mais rápida (10). As fibras A delta carreiam o impulso nervoso até o corno dorsal da medula espinhal (ME), onde o primeiro neurônio irá se conectar com as células marginais e, através de um interneurônio, com as células pedunculadas localizadas entre as lâminas I e II de Rexed. Estas últimas produzem metil-encefalina, que irá inibir a transmissão do impulso doloroso através das células da substância gelatinosa (SG) localizadas nas lâminas II e III de Rexed. Esse impulso é enviado também ao núcleo ventral do tálamo e depois ao córtex cerebral por meio do trato espinotalâmico, em que irá desencadear alterações que levam à ativação de um sistema descendente inibitório da dor, cujos principais neurotransmissores são a serotonina e a noradrenalina. Participam desse sistema, entre outros, a substância cinza periaqueductal, o núcleo arqueado do hipotálamo e o locus ceruleus. Já as fibras A beta, de rápida condução e responsáveis pelas sensações de tato e vibração, produzem inibição do impulso doloroso na SG do corno dorsal da ME através de um mecanismo no qual intervém a produção do ácido gama-aminobutírico (GABA). No entanto, com o avançar das pesquisas, descobriu-se que a acupuntura age também de outras formas, a saber (11): 
1. Liberação local de adenosina, óxido nítrico e peptídeo geneticamente relacionado à calcitonina (PGRC) no tecido periférico através dos reflexos axonal local e do corno dorsal da medula espinhal;

2. Modulação de tônus simpático e reflexos motores nos segmentos espinhais;

3. Modulação da atividade de sistemas descendentes inibitórios da dor partindo de regiões supraespinhais (tronco encefálico e outras);

4. Mudança na conectividade funcional do cérebro, com desativação de estruturas límbicas e modificações no eixo hipotálamo-hipófise-adrenal. Mudanças no córtex frontal, pré-frontal e somatossensorial;

5. Modulação do equilíbrio entre o sistema nervoso simpático e parassimpático;

6. Modulação da função do sistema imunológico;

7. Ativação do tecido conjuntivo e músculos, com liberação de miocinas, que possuem efeitos anti-inflamatórios locais diretos e indiretos, pela estimulação na produção de outras substâncias, como IL (interleucina) $1 \beta$ e IL-10.

No estudo de caso a seguir, relatamos o tratamento de uma paciente encaminhada ao ambulatório de Acupuntura da Universidade Federal Fluminense (UFF) para tratamento de tenossinovite de De Quervain, após falha de diversas outras formas de tratamento, e sua evolução alguns meses após encerrarmos o ciclo de tratamento, finalizando com as principais lições retiradas do caso. Cabe ressaltar que o ambulatório da UFF é um ambulatório-escola, contando com médicos pós-graduandos em Acupuntura e MTC da própria universidade e supervisionado pelos professores responsáveis pelo curso, todos com mais de 20 anos de prática de acupuntura. Funciona de segunda a sexta-feira, das $8 \mathrm{~h}$ às $17 \mathrm{~h}$. Os pacientes são referenciados pela rede pública do município de Niterói e por serviços os mais diversos da própria UFF, como ortopedia, reumatologia, neurologia etc. Os pacientes matriculados são inicialmente atendidos pelos médicos pós-graduandos e todos os casos são discutidos com os professores supervisores.

\section{Relato do Caso}

Paciente do sexo feminino, 54 anos, branca, professora, destra. Queixa principal: dor em antebraço, punho e mão direitos. Paciente referia dor na face radial do antebraço, punho e mão direitos há 6 meses, de intensidade moderada, grau 6 pela EVA (escala visual analógica da dor, varia de 0 a 10, portanto 6 significa dor de grau moderado). A dor piorava ao mover a mão direita, principalmente no movimento de pinçamento do polegar. Como utiliza as mãos como instrumento de trabalho, isso a estava incomodando muito. A dor melhorava um pouco com calor local.

História patológica pregressa, familiar e social não apresentavam nada digno de nota.

Ao exame físico do punho direito, dor à pressão no local, próximo ao processo estiloide do rádio, e testes de Finkelstein e Eichoff positivos.

Fez uso de AINES (anti-inflamatórios não esteroides), com melhora temporária, apenas quando em uso da medicação. Seu clínico geral a encaminhou ao ortopedista, que novamente prescreveu AINES e fisioterapia. Paciente retornou 3 meses depois sem alívio da dor. Foi então realizada infiltração local com corticoide com melhora parcial. $\mathrm{O}$ ortopedista indicou cirurgia como tratamento de cura para o caso. A paciente, amedrontada pela possibilidade de cirurgia, foi encaminhada para acupuntura. Na primeira consulta no ambulatório de acupuntura, paciente apresenta-se com dor, tensa, ansiosa, com medo de agulhas.

Ao exame pela MTC, notava-se um pulso superficial e tenso com uma língua rosada, úmida, com saburra branca. 
Pelos critérios de diagnóstico sindrômico da MTC (6), fícou claro tratar-se de caso com síndrome de obstrução dolorosa do tipo frio (Tong Bi), pois, nesse tipo de síndrome, a dor costuma ser fixa, mais intensa e alivia com a aplicação de calor local. Ainda de acordo com a MTC, a dor aguda, nesses casos, é causada pela circulação lenta de $Q i$ (energia) e de Xue (sangue) nos meridianos, devido ao excesso de frio. O frio tem efeito estagnante sobre o $Q i$ e sobre o Xue. O revestimento branco da língua e o pulso tenso são sinais de frio patogênico e dor.

Em geral, nos casos da tenossinovite de De Quervain, os meridianos afetados são os do Pulmão (Fei) e do Intestino Grosso (Da Chang) (7). No caso dessa paciente, o meridiano mais afetado parecia ser o do Intestino Grosso, que segue um trajeto muito semelhante aos locais da dor referida pela paciente.

Nesse momento, optamos por oferecer o tratamento preconizado pela MTC nesses casos, cujo princípio é eliminar o frio, aquecendo os meridianos afetados e promover a circulação do $Q i$ e do Xue. Escolhemos utilizar as seguintes estratégias, num total de 5 (cinco) sessões, com intervalo de uma semana entre as mesmas:

- Pontos locais: Intestino Grosso 4 e 5 (IG4 e IG5), Pulmão 7 (P7), pontos dolorosos locais. Estes últimos se localizavam sobre ou próximos aos músculos abdutor longo e extensor curto do polegar;

- Pontos para ansiedade: Fígado 3 (F3) e Yintang (ponto extra entre as sobrancelhas). Esses dois pontos tiveram como objetivo combater a ansiedade;

- Em duas sessões, associamos o tratamento com o moxibustão, que é a erva de artemísia, local, em combustão, por alguns segundos. Utilizamos a moxa nas duas primeiras sessões, pois sabe-se de seu efeito salutar na dispersão do frio patogênico. Depois, não mais utilizamos a moxa porque não julgamos necessário, uma vez que paciente vinha melhorando muito, ficando quase assintomática já a partir da $2^{a}$ sessão;

A localização dos pontos utilizados está descrita na tabela abaixo.

Tabela 1: Principais pontos utilizados no tratamento

\begin{tabular}{l|l} 
Ponto & Localização \\
\hline IG4 (Hegu) & $\begin{array}{l}\text { Situa-se na metade do } 2^{\circ} \text { metacarpo, entre o } 1^{\circ} \text { e o } 2^{\circ} \text { ossos metacarpos, sobre } \\
\text { a saliência muscular quando se faz a adução do polegar }\end{array}$ \\
\hline IG5 (Yangxi) & $\begin{array}{l}\text { Situa-se em uma reentrância no fundo da tabaqueira anatômica, na prega do } \\
\text { dorso do punho }\end{array}$ \\
\hline P7 (Lieque) & $\begin{array}{l}\text { Situa-se de um a um e meio tsun proximais à prega ventral do punho, } \\
\text { lateralmente à artéria radial, entre os tendões dos músculos braquiorradial e } \\
\text { abdutor longo do polegar. }\end{array}$ \\
\hline F3 (Taichong) & $\begin{array}{l}\text { Situa-se no dorso do pé, entre o primeiro e segundo ossos do metatarso, de um } \\
\text { a um e meio tsun proximal à prega interdigital. }\end{array}$ \\
\hline Ex-HN3 (Yintang) & $\begin{array}{l}\text { Situa-se na linha mediana anterior da face, na correspondência de uma linha } \\
\text { horizontal, unindo as extremidades mediais das sobrancelhas. }\end{array}$
\end{tabular}

Os pontos foram agulhados com agulhas de aço inoxidável Dong Bang 0,25 mm x 40 mm (diâmetro x comprimento), de uso único, estéreis, que eram deixadas no local por 20 minutos. Mesmo nas sessões nas quais foi utilizada a moxa, o tempo de permanência das agulhas foi o mesmo. Não foi realizada manipulação das agulhas durante o procedimento e o sentido de colocação das agulhas foi perpendicular, com exceção do Yintang, cuja inserção foi oblíqua, dirigida para baixo, e do P7, cuja inserção foi oblíqua, em direção ao punho. As Figuras 2 e 3 mostram os pontos utilizados. 


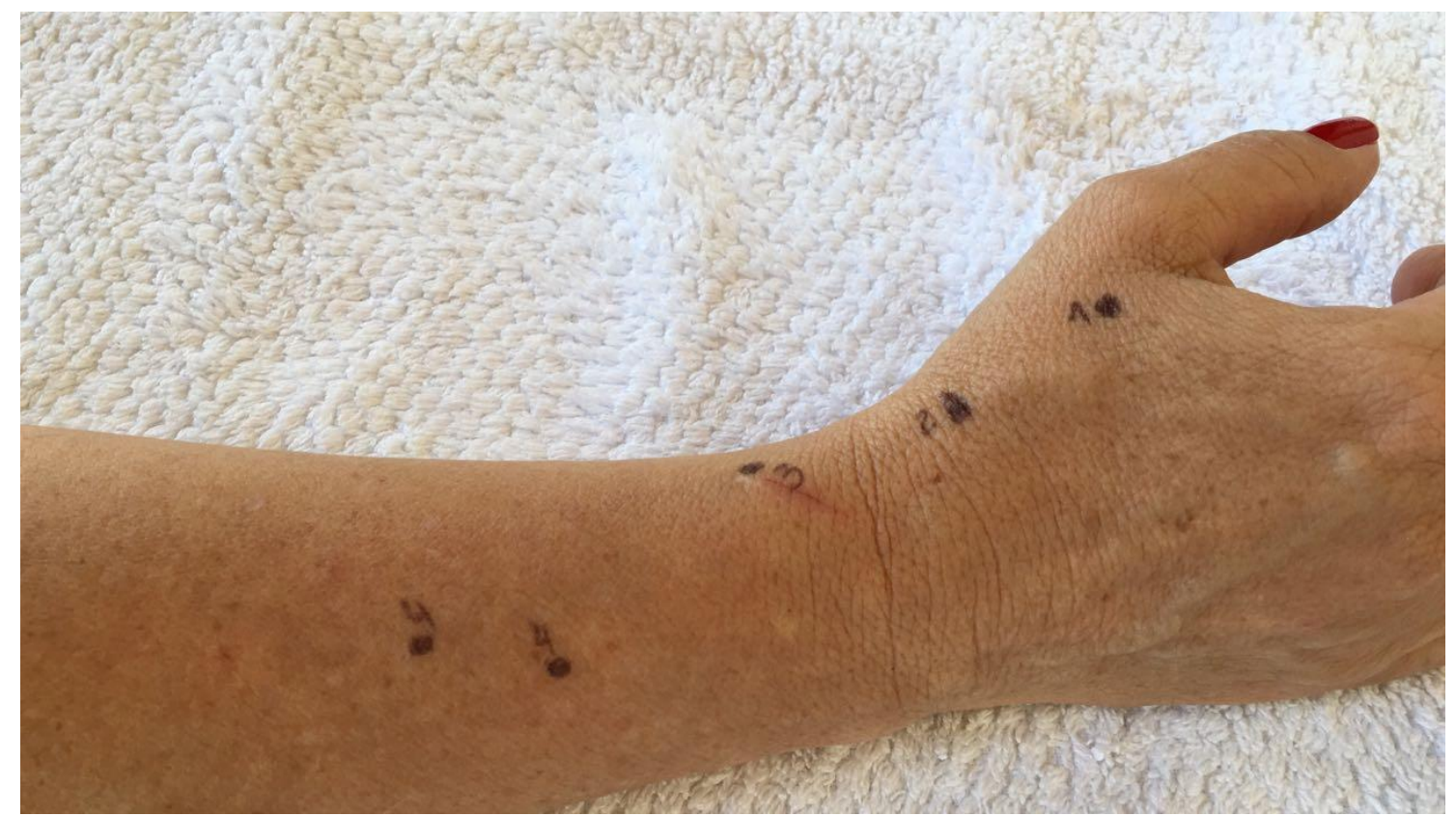

Figura 2: 1. IG4; 2. IG5, 3. P7; 4. Pontos Ah shi, encontrados em algumas sessões.
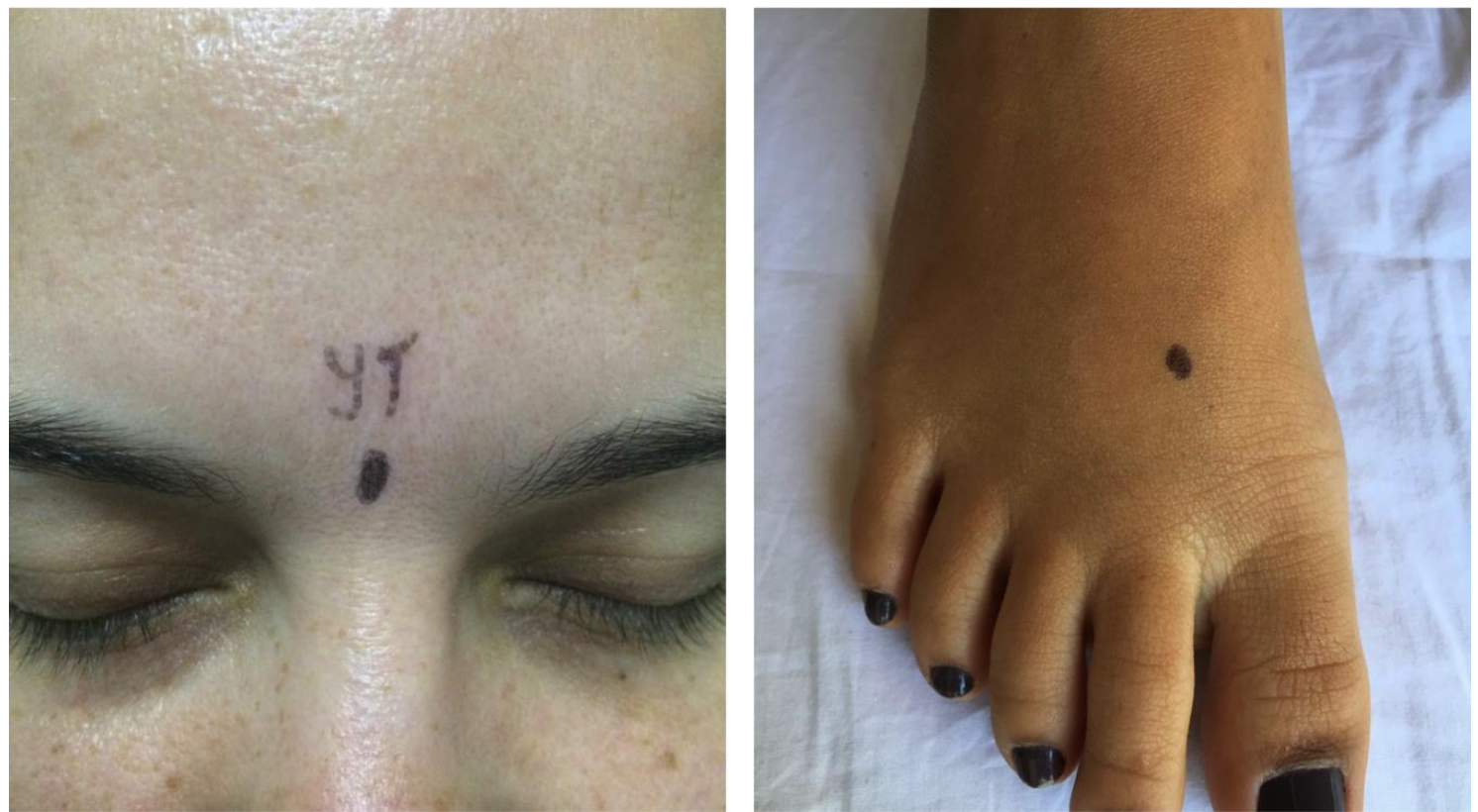

Figura 3: Yintang e F3.

Logo após a primeira sessão de tratamento, a paciente já apresentou melhora expressiva, tendo saído do ambulatório sem dor. À medida que as sessões foram prosseguindo, essa melhora foi se consolidando, sendo que da $4^{a}$ para a $5^{\mathrm{a}}$ sessão a paciente não mais apresentou dor no punho.

Revimos a paciente 2 (duas) semanas depois, ela continuava assintomática, o que se manteve mesmo após 3 meses depois da última sessão de acupuntura. Desde então, não mais tivemos contato com a paciente. No entanto, a paciente foi instruída, quando de sua alta, a nos procurar caso voltasse a apresentar algum sintoma. Apesar de reconhecermos a importância de saber a evolução em médio e longo prazos de casos como esse, não é rotina de nosso ambulatório programar retorno de pacientes que estejam assintomáticos. 


\section{Considerações finais}

Trata-se de caso simples, relativamente comum de aparecer no dia a dia de um ambulatório de acupuntura. Foi-nos possível, utilizando uma racionalidade médica diferente daquela aprendida no Ocidente, fornecer um tratamento altamente resolutivo para a paciente em questão, devolvendo-a às suas atividades diárias, incluindo seu trabalho.

Em estudo randomizado recentemente publicado (12), comparando-se a acupuntura com a injeção de corticoide local para tratamento da tenossinovite de De Quervain, envolvendo 30 pacientes randomizados para dois grupos, grupo injeção $(n=15)$ versus grupo acupuntura $(\mathrm{n}=15)$, notou-se benefício semelhante entre os dois tratamentos ao longo de 6 semanas de evolução, sem diferenças estatisticamente significativas entre os grupos após 6 semanas, em relação à escala de dor e ao grau de incapacidade para o trabalho com o membro. Apesar de a injeção local de corticoide ser ainda o tratamento padrão para esse tipo de problema, a acupuntura surge como alternativa válida e muito importante, sem os potenciais efeitos deletérios da droga, além de ter a vantagem de poder ser repetida várias vezes sem causar dano. Além disso, é uma terapia de baixo custo e praticamente desprovida de efeitos colaterais, o que não é o caso dos medicamentos anti-inflamatórios, em especial os corticoides. No caso de nossa paciente, a infiltração de corticoide local resultou em benefício de curta duração, tendo os sintomas retornado em prazo de tempo menor do que 2 semanas, motivo pelo qual a paciente foi encaminhada para acupuntura.

Outro artigo, publicado em 2014 (13), propõe que sejam associados aos pontos clássicos de acupuntura, na tenossinovite de De Quervain, pontos correspondentes aos músculos acometidos nessa patologia, o abdutor longo e o extensor curto do polegar, o que propiciaria melhora mais rápida, uma vez que a tendinopatia é, em geral, resultado de uma lesão secundária causada por um problema muscular (14). Em nossa paciente, os pontos dolorosos encontrados estavam no trajeto ou próximos a esses músculos, corroborando a impressão dos autores que propõem essa estratégia e certamente tendo contribuído para a melhora mais rápida e duradoura que obtivemos.

É sempre interessante raciocinar em relação aos mecanismos de ação através dos quais a acupuntura atuou nesse caso. Nesse aspecto, podemos encarar de duas formas: 1) sob a ótica da MTC; 2) do ponto de vista da medicina ocidental. Sob a ótica da MTC, já havia sido anteriormente mencionado que esse caso pode ser entendido como uma síndrome de obstrução dolorosa tipo frio, acometendo os meridianos do Intestino Grosso e Pulmão. Nesse caso, o frio promove estagnação de $Q i$ e Xue (Sangue) nos meridianos afetados (7). Entendemos que a acupuntura atua eliminando o fator patogênico (Frio), aquecendo os meridianos afetados e promovendo a circulação de $Q i$ e Xue nos mesmos. Do ponto de vista ocidental, a atuação da acupuntura se faz em nível local, segmentar, suprassegmentar e cortical (11). Em nível local, ocorre a produção de substâncias anti-inflamatórias, como as interleucinas $1 \beta$ e 10, além do PGRC, que aumenta o fluxo sanguíneo local, diminuindo, com isso, a inflamação. A ação segmentar ocorre no corno dorsal da medula espinhal, onde impulsos carreados pelas fibras A delta irão se conectar com as células pedunculadas na lâmina II de Rexed e estimularão a produção de metil-encefalina, que, por sua vez, inibe a transmissão do impulso doloroso pelas células da SG. As ações suprassegmentar e cortical colocam em jogo inúmeros mecanismos já mencionados anteriormente, das quais participam o tálamo, a substância cinza periaqueductal, o núcleo arqueado do hipotálamo, além de áreas corticais superiores. Os principais neurotransmissores desse sistema descendente inibitório da dor são a serotonina e a noradrenalina. 
Concluindo, podemos dizer que esse caso nos ensina que muitas alternativas não medicamentosas e menos agressivas são possíveis no tratamento da dor decorrente de processos inflamatórios como a tenossinovite de De Quervain, despontando a acupuntura como opção válida, custo-efetiva e eficaz dentro do cenário das práticas integrativas e complementares, com inúmeros estudos já publicados, comprovando seus efeitos em curto e médio prazo.

\section{Referências}

1. De Quervain F. On the nature and treatment of stenosing tendovaginitis on the styloid process of the radius. (Translated article: Muenchener Medizinische Wochenschrift 1912; 59:5-6). J Hand Surg Br 2005; 30:392-4.

2. Arend CF. Tenossinovite e sinovite do primeiro compartimento extensor do punho: o que o ultrassonografista precisa saber. Radiol Bras. 2012;45(4):219-224.

3. Snider RK. Tratamento das doenças do sistema musculoesquelético. $1^{\mathrm{a}}$ ed. São Paulo: Editora Manole; 2000.

4. Finkelstein H. Stenosing tenovaginitis at the radial styloid process. J Bone Joint Surg. 1930; 12:509-40.

5. Eichoff E. Pathogenese der Tendovaginitis stenosans. Bruns' Beitrage Z Klin Chir. 1927; CXXXIX:746.

6. Wang LG. Tratado Contemporâneo de Acupuntura e Moxibustão. São Paulo: CEIMEC; 2005.

7. Yamamura Y. Acupuntura tradicional: a arte de inserir. São Paulo: Editora Roca; 2003.

8. Li X, Wang R, Xing X, Shi X, Tian J, Zhang J et al. Acupuncture for Myofascial Pain Syndrome: A Network Meta-Analysis of 33 Randomized Controlled Trials. Pain Physician. 2017;20(6):E883E902.

9. NIH. NIH Consensus Conference. Acupuncture. JAMA. 1998; 280 (17):1518-24.

10. Bowsher D. Mechanisms of Acupuncture. In: Medical Acupuncture - A Western Scientific Approach. $1^{\text {st }}$ ed. Edinburgh: Churchill Livingstone, 1998. p. 69-82.

11. Filshie J, White A. Cummings M. Medical Acupuncture - A Western Scientific Approach. $2^{\text {nd }}$ ed. London: Elsevier; 2016.

12. Hadianfard M, Ashraf A, Fakheri M, Nasiri A. Efficacy of Acupuncture versus Local Methylprednisolone Acetate Injection in De Quervain's Tenosynovitis: A Randomized Controlled Trial. J Acupunct Meridian Stud. 2014; 7(3):115-21.

13. Silva JBG da, Batigália F. Acupuncture in De Quervain's disease: a treatment proposal. Acupunct Med. 2014; 32(1):70-2.

14. Neal BS, Longbottom J. Is there a role for acupuncture in the treatment of tendinopathy? Acupunct Med. 2012; 30:346-9. 